\title{
Erratum to: Cluster categorization of urban roads to optimize their noise monitoring
}

\author{
G. Zambon • R. Benocci • G. Brambilla
}

Published online: 13 February 2016

(C) Springer International Publishing Switzerland 2016

\section{Erratum to: Environ Monit Assess}

DOI 10.1007/s10661-015-4994-4

The original article contained an error.

The (=) symbol in equation 4 was written as (-) in PDF version. However, it has been addressed and is not anymore present in the published copies of the article.

The corrected Equation 4 is shown below.

$L_{\text {Aeqd }}=10 \times\left[\frac{1}{16} \sum_{i=1}^{16} 10^{0.1 L_{\text {Aeqhi }}}\right][\mathrm{dB}(\mathrm{A})]$

The online version of the original article can be found at http://dx. doi.org/10.1007/s10661-015-4994-4.

G. Zambon $(\bowtie) \cdot R$. Benocci

Department of Earth and Environmental Sciences, Università degli Studi di Milano, Bicocca, Piazza dalla Scienza 1, 20126 Milan, Italy

e-mail: giovanni.zambon@unimib.it

G. Brambilla

Istituto di Acustica e Sensoristica "Orso Mario Corbino", CNR,

Via del Fosso del Cavaliere 100, 00133 Rome, Italy 